ARTICLE

Received 2 Jul 2012 | Accepted 2 Jan 2013 | Published 12 Feb 2013 DOl: 10.1038/ncomms2428

\title{
Persistent high-energy spin excitations in iron-pnictide superconductors
}

Ke-Jin Zhou ${ }^{1,2}$, Yao-Bo Huang ${ }^{1,3}$, Claude Monney ${ }^{1}, X_{i}$ Dai ${ }^{3}$, Vladimir N. Strocov', Nan-Lin Wang ${ }^{3}$, Zhi-Guo Chen ${ }^{3}$, Chenglin Zhang ${ }^{4}$, Pengcheng Dai ${ }^{3,4}$, Luc Patthey ${ }^{1,6}$, Jeroen van den Brink ${ }^{5}$, Hong Ding $^{3} \&$ Thorsten Schmitt ${ }^{1}$

Motivated by the premise that superconductivity in iron-based superconductors is unconventional and mediated by spin fluctuations, an intense research effort has been focused on characterizing the spin-excitation spectrum in the magnetically ordered parent phases of the Fe pnictides and chalcogenides. For these undoped materials, it is well established that the spin-excitation spectrum consists of sharp, highly dispersive magnons. The fate of these highenergy magnetic modes upon sizable doping with holes is hitherto unresolved. Here we demonstrate, using resonant inelastic $\mathrm{X}$-ray scattering, that optimally hole-doped superconducting $\mathrm{Ba}_{0.6} \mathrm{~K}_{0.4} \mathrm{Fe}_{2} \mathrm{As}_{2}$ retains well-defined, dispersive high-energy modes of magnetic origin. These paramagnon modes are softer than, though as intense as, the magnons of undoped antiferromagnetic $\mathrm{BaFe}_{2} \mathrm{As}_{2}$. The persistence of spin excitations well into the superconducting phase suggests that the spin fluctuations in Fe-pnictide superconductors originate from a distinctly correlated spin state. This connects Fe pnictides to cuprates, for which, in spite of fundamental electronic structure differences, similar paramagnons are present.

\footnotetext{
${ }^{1}$ Paul Scherrer Institut, Swiss Light Source, Villigen PSI CH-5232, Switzerland. ${ }^{2}$ Diamond Light Source, Harwell Science and Innovation Campus, Didcot, Oxfordshire OX11 ODE, UK. ${ }^{3}$ Beijing National Laboratory for Condensed Matter Physics, and Institute of Physics, Chinese Academy of Sciences, Beijing 100190, China. ${ }^{4}$ Department of Physics and Astronomy, The University of Tennessee, Knoxville, Tennessee 37996, USA. ${ }^{5}$ Institute for Theoretical Solid State Physics, IFW Dresden, Dresden, 01171, Germany. 6 Paul Scherrer Institut, SwissFEL, Villigen PSI, CH-5232, Switzerland. Correspondence and requests for materials should be addressed to K.J.Z. (email: kejin.zhou@diamond.ac.uk) or to T.S. (thorsten.schmitt@psi.ch).
} 
$\mathrm{O}$ ne of the greatest challenges posed by the recently discovered Fe-pnictide superconductors ${ }^{1}$ is to identify the mechanism by which electrons pair when they condense into the superconducting state. Phonon-driven pairing, as for conventional superconductors, is counter-indicated by theoretical calculations $s^{2}$ and the electron-phonon interaction is deemed too weak to directly account for the observed high critical temperatures of these materials. Phonon-induced orbital fluctuations were suggested to be responsible for the observed high critical temperatures $\mathrm{T}_{\mathcal{c}}$, though no consensus on this has been reached so far ${ }^{3,4}$. As superconductivity emerges for $\mathrm{Fe}$ pnictides in the vicinity of an antiferromagnetic ( $\mathrm{AF}$ ) ordered phase likewise as for cuprates, particular attention has, therefore, been given to spin fluctuations that emerge from the interactions between electron $\operatorname{spins}^{5-10}$. For these to become operative in pairing electrons and to induce superconductivity, first the longrange magnetic ordering-the spin-density wave phase that is stable in the parent Fe pnictides-has to melt. The subsequent crucial question is how well defined the resulting spin fluctuations are, which directly relates to the strength of magnetic short-range order and coherence of its magnetic excitations.

In cuprates, for which magnetic properties are governed by strong superexchange interactions between localized spin-1/2 moments in a single $\mathrm{Cu} 3 d_{\mathrm{x} 2-\mathrm{y} 2}$ orbital, doping-induced melting of the AF ordered phase gives way to a highly correlated spinliquid state. This spin-liquid carries well-defined high-energy paramagnon modes, present also in optimally doped systems ${ }^{11}$. For the metallic Fe pnictides, a central debate of the field is whether the occurring magnetic instability is due to a nesting of hole and electron Fermi-surface pockets (weak coupling scenario), for which weak exchange interactions are enough to create long-range magnetic ordering ${ }^{12,13}$. An alternative view is describing the magnetism of the parent $\mathrm{Fe}$ pnictides by a correlated spin state with large localized fluctuating magnetic moments having the key role (strong coupling scenario) $)^{7,14,15}$.

In the present study with resonant inelastic X-ray scattering (RIXS) at the $\mathrm{Fe}_{3}$ edge, we establish in optimally hole-doped $\mathrm{Ba}_{0.6} \mathrm{~K}_{0.4} \mathrm{Fe}_{2} \mathrm{As}_{2}$ (BKFA) the presence of well-defined and dispersive high-energy paramagnons. Their spectral intensity and width are comparable to the magnons of undoped $\mathrm{AF}$ $\mathrm{BaFe}_{2} \mathrm{As}_{2}$ (BFA), reaching the energy of $150 \mathrm{meV}$ at the Brioullin zone (BZ) edges. These paramagnon peaks reveal the presence of a distinctly correlated spin state in BKFA. Its existence connects the Fe-pnictide superconductors conceptually to the high- $T_{\mathrm{c}}$ cuprates, where superconductivity also emerges in the presence of a pronounced paramagnon background ${ }^{11}$.

\section{Results}

Dispersive high-energy magnons in parent BFA. To measure spin excitations in parent BFA and superconducting BKFA Fepnictide samples, we employ RIXS, which has recently been established as a powerful probe of the dispersion of magnetic excitations in a wide energy-momentum window. Many studies on undoped parent cuprates have demonstrated the sensitivity of RIXS to single-magnon excitations ${ }^{11,16-21}$ and collective orbital excitations ${ }^{1 f}$. Doped superconducting cuprates exhibit intense paramagnons, damped spin excitations over much of the $\mathrm{BZ}$ with dispersions and spectral weights closely similar to those of magnons in undoped AF ordered parent systems ${ }^{11,19}$.

The BFA and BKFA samples used in our RIXS experiments are single crystals grown using the self-flux method ${ }^{22,23}$. Resistivity and bulk magnetic susceptibility measurements demonstrate the high quality of all samples (see Supplementary Fig. S1). Figure 1a,b displays the schematics of the RIXS experimental geometry, as well as the reciprocal space that can be reached with
Fe $\mathrm{L}_{3}$ RIXS. A typical Fe $\mathrm{L}_{3}$ edge X-ray absorption spectrum of BFA is shown in Fig. 1c, in good agreement with a previous report $^{24}$. In Fig. 1d,e, a set of momentum-resolved $\mathrm{Fe} \mathrm{L}_{3}$ RIXS spectra of BFA using $\pi$ polarized incoming light at $(0,0),(0.5,0)$ and $(0.35,0.35)$ in the $\mathrm{BZ}$ are displayed. All these spectra exhibit intense $\mathrm{Fe} 3 \mathrm{~d}$ fluorescence at around $-2 \mathrm{eV}$ energy transfer, which has been observed in RIXS studies on other Fe pnictides ${ }^{24}$ and chalcogenides ${ }^{25}$. In addition to these previous investigations $^{24,25}$, we reveal near the $\mathrm{BZ}$ edges well-defined momentum dispersive excitations centered around $200 \mathrm{meV}$ next to the quasi-elastic peak in the vicinity of zero energy.

In Fig. 2a,b, we show two sets of $\mathrm{Fe} \mathrm{L}_{3}$ RIXS spectra of BFA with the momentum transfer directed along two high-symmetry directions, $(0,0)-(1,0)$ and $(0,0)-(1,1)$. All RIXS spectra for both BZ directions display well-defined excitations within an energy range of $0-300 \mathrm{meV}$ superimposed on the tail of the Fe $3 \mathrm{~d}$ fluorescence. For high-momentum transfer $\mathrm{q}_{/ /}$, these excitations clearly separate from the quasi-elastic peak. Approaching the $\Gamma$ point, the excitation intensity decreases and the energy position shifts towards the quasi-elastic peak. To quantitatively analyze these excitations, we subtract the fluorescence background employing the method introduced in Hancock et al..$^{25}$ and decompose the spectral response close to the quasi-elastic peak (see Supplementary Fig. S2). As demonstrated in Fig. 2c, the excitation at the zone boundary peaks at around $200 \mathrm{meV}$ and contains a high-energy tail.

In Fig. 2d,e, we show that the corresponding sets of excitations clearly disperse as a function of transferred momentum after subtraction of background and quasi-elastic peak. For the RIXS spectra excited with $\sigma$ polarized incoming light, the excitation intensity is slightly suppressed. Furthermore, the spectral weight is almost quenched when the incident energy moves away from the $\mathrm{Fe}_{3}$ resonance (see Supplementary Fig. S3). This observation is representative for single-magnon excitations as revealed with $\mathrm{Cu} \mathrm{L}_{3}$ RIXS for many cuprates ${ }^{11,16-19}$. Unlike the parent cuprates, which are long-range ordered AF Mott insulators, the Fe-pnictide parent compounds are AF ordered spin-density wave semi-metals with compensating electron- and hole-like Fermi-surface pockets involving several $\mathrm{Fe} 3 \mathrm{~d}$ orbitals. Thus, charge excitations (electron-hole pair excitations) can fall in the same energy window as spin excitation ${ }^{26}$. However, the electron-hole continuum is expected to be temperature independent ${ }^{27}$. In contrast, our RIXS measurements clearly revealed that the sharp excitations in the AF phase become much less well defined in the paramagnetic phase, thereby strongly suggesting the magnetic origin of these excitations (see Supplementary Fig. S4).

Comparison of our RIXS data for the parent BFA with available inelastic neutron scattering (INS) results clearly shows that the inelastic X-ray response is dominated by magnetic excitations, which is not unexpected as in direct RIXS spin-flip scattering is strong ${ }^{28,29}$. Fits to the inelastic response with an asymmetrical Lorentzian line shape convoluted with a Gaussian resolution function give a good description of the data ${ }^{11}$ (see Supplementary Fig. S2). In Fig. 2f, we plot the dispersion of the RIXS peak energy position as a function of momentum transfer. To exclude the effect of sample-dependent variations, we confirmed these results with independent measurements on additional samples. On top of RIXS peak positions, we overlay the dispersion curve of spin excitations extracted from INS measurements on a BFA parent sample ${ }^{30}$ (see Supplementary Discussion). The excellent agreement between INS and our RIXS data indicates on a simple phenomenological basis that indeed the dispersing excitations in the inelastic response are of magnetic origin. This conclusion is further supported by the fact that the line shape of the excitations can be well fitted using the formula describing the imaginary part of the dynamical spin susceptibility. 
a

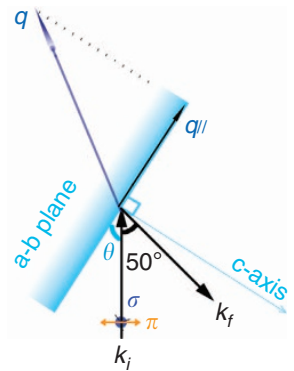

b

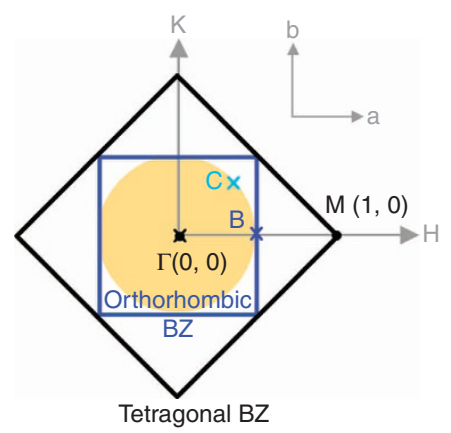

Tetragonal BZ

C

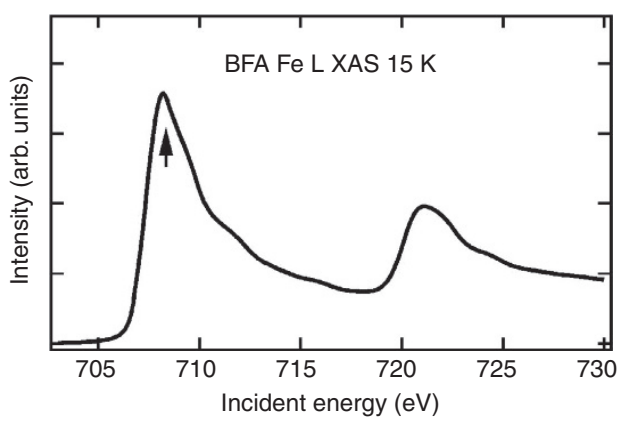

d

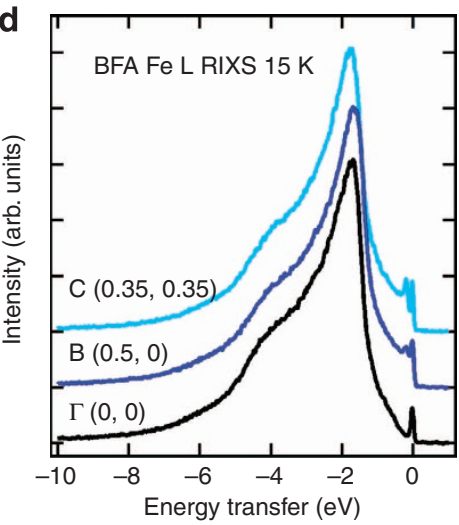

e

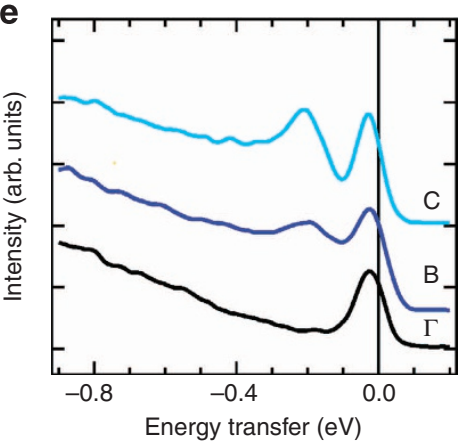

Figure 1 | Fe L X-ray absorption spectrum (XAS) and RIXS spectra of BFA. (a) Schematics of RIXS $130^{\circ}$ back-scattering geometry with an included angle of $50^{\circ}$ between the incoming and outgoing light vectors, $\mathbf{k}_{\mathbf{i}}$ and $\mathbf{k}_{\mathbf{f}}$, respectively. The sample a-b plane lies perpendicular to the scattering plane. $q / /$ is the projection of the momentum transfer $\mathbf{q}$ along the a-b plane. The incoming light is polarized either parallel $(\pi)$ or perpendicular $(\sigma)$ to the scattering plane with a grazing incident angle $(\theta)$. (b) Schematic view of the reciprocal space which can be covered by $F e L_{3}$ RIXS shaded by a yellow circle. $\Gamma, B$ and $C$ in Fig. 1d,e are the reciprocal space positions at which RIXS spectra were collected. Black (blue) squares represent the tetragonal (orthorhombic) Brillouin zone (BZ). All RIXS spectra use the orthorhombic BZ convention for defining relative momentum transfer values. $\Gamma$ point is the structural zone center, while $\Gamma M$ is the AF ordering wave vector. (c) A representative Fe L XAS of BFA collected with $\pi$ polarized incoming light at $15 \mathrm{~K}$. The arrow denotes the fixed incident energy of the Fe $\mathrm{L}_{3}$ resonance for all RIXS spectra. (d) Three typical RIXS spectra of BFA collected at $15 \mathrm{~K}$ with $\pi$ polarized incoming light, at $\left(q_{x}, q_{y}\right)=(0,0)(\Gamma),(0.5,0)(B)$ and $(0.35,0.35)(C)$ using relative lattice units. The relation $(H, K)=\left(q_{x} a / 2 \pi, q_{y} b / 2 \pi\right)$ is adopted where $a=5.62 \AA$ and $b=5.57 \AA$ are the orthorhombic unit cell lattice parameters in the spin-density wave phase. (e) Zoom into the low-energy region of Fig. 1d.

Closer comparison with the INS study on parent $\mathrm{BFA}^{30}$ demonstrates, furthermore, that our RIXS data show comparable half width at half maximum (HWHM) of the spin excitations, that is, damping (around $100 \mathrm{meV}$ ) at the zone boundary (see Fig. 4b).

Persistent high-energy paramagnons in optimally hole-doped BKFA. Having demonstrated that $\mathrm{Fe}_{3}$ RIXS allows to measure the dispersion of spin excitations in the AF ordered state, we are well prepared to further explore how spin excitations evolve in the superconducting (SC) phase. We focus now on an optimally hole-doped BKFA superconductor $\left(T_{c}=39 \mathrm{~K}\right)$, for which highenergy spin excitations have not been reported so far. By performing the same measurements as for BFA, the two corresponding sets of RIXS spectra along $(0,0)-(1,0)$ and $(0,0)-(1,1)$ BZ directions of BKFA are obtained and displayed in Fig. 3a,b. Remarkably, similar to BFA, BKFA also shows pronounced and well-defined excitations persisting up to $150 \mathrm{meV}$. Because these follow the same polarization and incident energy dependences as parent BFA and appear as smoothly connected to the magnetic modes in BFA, we conclude that these excitations in BKFA are likewise of magnetic origin. These paramagnon excitations show clear dispersion along the two high-symmetry directions after subtraction of background and quasi-elastic peak (see Fig. 3c). For fitting of the spectral profile, the same function as for parent
BFA was used. Fitted peak energy, HWHM, and integrated spectral weight as a function of momentum transfer are summarized for parent BFA and superconducting BKFA in Fig. 4.

To establish whether or not the observed spin excitations are exclusively linked to the system being in its SC state, we further carried out measurements in the normal state of BKFA. At $60 \mathrm{~K}$, above the SC transition temperature of $39 \mathrm{~K}$, paramagnon excitations are still well defined and comparable to the SC phase without losing intensity (see Supplementary Fig. S6). We note that a temperature-dependent INS study on electron-doped $\mathrm{Ba}\left(\mathrm{Fe}_{1-\mathrm{x}} \mathrm{Co}_{\mathrm{x}}\right)_{2} \mathrm{As}_{2}(x=0.074)$ is consistent with our observation that the spin excitations remain unaltered across the $T_{\mathrm{c}}$ (ref. 31 ).

\section{Discussion}

From the comparison in Fig. 4a, it is noticeable that the spinexcitation energies in BKFA get softened relative to the ones in BFA. Softening of spin excitations upon doping has been observed in cuprates both in RIXS ${ }^{11}$ and INS $^{32}$ studies. Moreover, doping induces damping of the spin excitations in cuprates because of the interaction with electron-hole excitations. Interestingly, doping of parent BFA does not create visible further damping of spin excitations (Fig. 4b). The line width of around $100 \mathrm{meV}$ HWHM is likely intrinsic, because it is nearly two and half times the total instrumental resolution of our RIXS experiment (HWHM $\sim 40 \mathrm{meV}$ ). The observed large broadening 

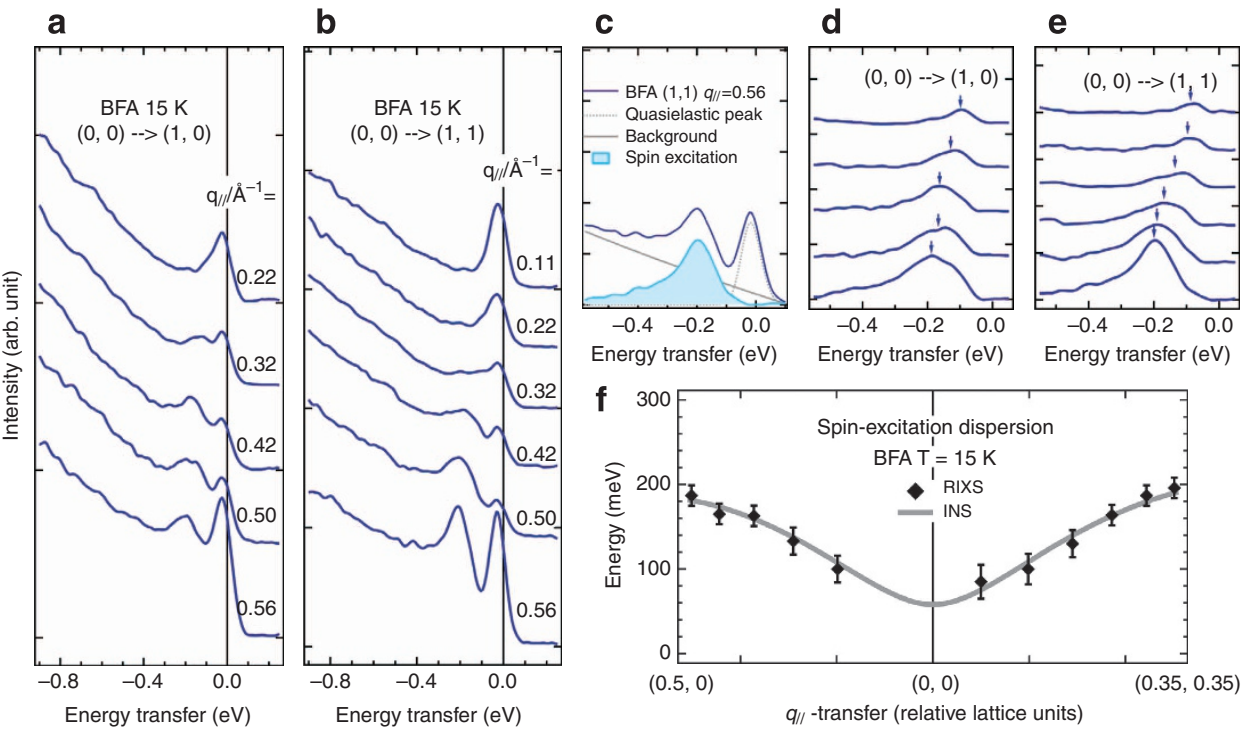

Figure 2 I Spin excitations of parent BFA. (a,b) RIXS spectra of parent BFA along $(0,0)-(1,0)$ and $(0,0)-(1,1)$ directions in the first Brillouin zone obtained at $15 \mathrm{~K}$ with $\pi$ polarized incoming light. The numbers displayed at the right side of each spectrum denote the absolute wave vector $q_{/ / /}$projected along the sample a-b plane (as in $\mathbf{e}$ and Fig. 3a-c). (c) A representative spectrum at the BZ edge demonstrating the subtraction of the fluorescence background and the quasi-elastic peak in order to obtain the spin-excitation component. (d-e) Spin excitations along $(0,0)-(1,0)($ left $)$ and $(0,0)-(1,1)$ (right) directions after subtracting the background and quasi-elastic peak. The arrows mark the fitted peak centers. (f) Spin-excitation dispersions along $(0,0)-(1,0)$ (left) and $(0,0)-(1,1)$ (right) directions. $q_{/ /}$transfer is re-scaled to relative lattice units. The filled black diamonds are fitted energy positions from the data shown in $\mathbf{d}$ and $\mathbf{e}$. Vertical bars represent errors from the fitting of the energy position of the spin excitations. The thick grey line is the calculated dispersion curve using the effective superexchange parameters from INS data ${ }^{30}$ (see Methods).
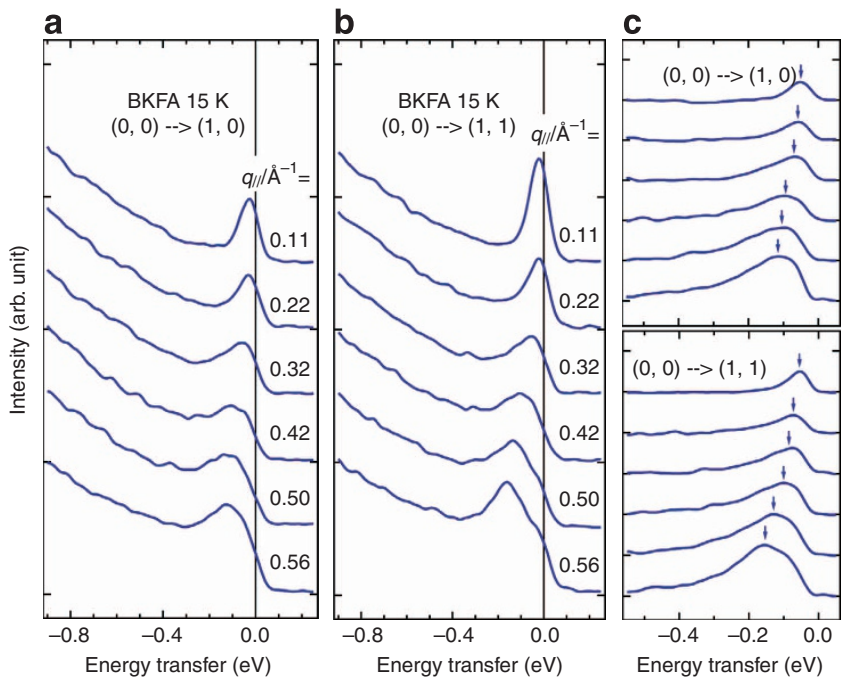

Figure 3 | Spin excitations of the optimally hole-doped BKFA superconductor. (a,b) RIXS spectra of BKFA along $(0,0)-(1,0)$ and $(0,0)-(1,1)$ directions obtained at $15 \mathrm{~K}$ with $\pi$ polarized incoming light. The probed $q_{/ /}$range is the same as in the parent BFA sample. (c) Spin excitations along $(0,0)-(1,0)$ (upper panel) and $(0,0)-(1,1)$ (lower panel) directions at $15 \mathrm{~K}$ after subtracting the background and quasi-elastic peak. The arrows mark the fitted peak centers. Spectra are vertically stacked according to different $q_{/ /}$values.

of spin excitations already in the parent pnictide differs significantly from the situation of the parent cuprates $^{11,20}$. In the latter case, the RIXS instrumental resolution defines the single-magnon line width because of the long magnon lifetime. The larger observed magnon line width in the parent Fe pnictide is, however, not unexpected because its spin excitations, despite being well defined, are essentially damped by the interaction with itinerant electrons due to its metallic nature ${ }^{9,30,33}$. Carrier doping into the SC state does not necessarily add further damping of spin excitations, consistent with our RIXS observation (Fig. 4b). Such different damping behaviour of the spin excitations demonstrates that Fe pnictides indeed deviate in this point from the strongly localized antiferromagnetism in cuprates, which can be ideally described by the Heisenberg model. We further notice that the total spin-excitation spectral weight is largely preserved when crossing from the AF to the SC phase (Fig. 4c). The same effect has been discovered in a RIXS study on the $\mathrm{YBa}_{2} \mathrm{Cu}_{3} \mathrm{O}_{7-x}$ family, where well-defined dispersive paramagnons are also present and their integrated spectral weight persist in parent, under- and slightly overdoped compounds ${ }^{11}$. Although pnictides and 

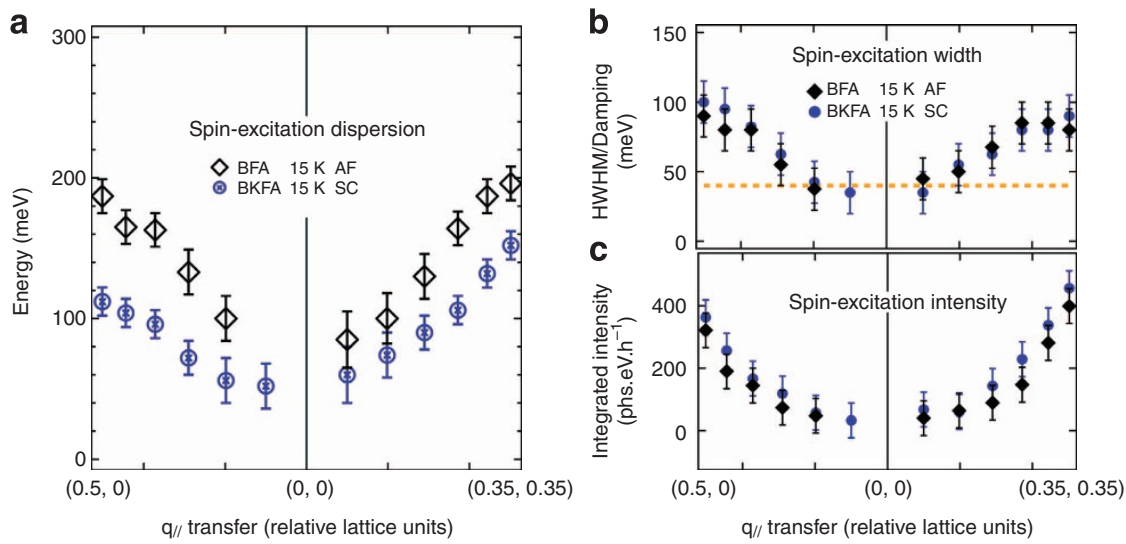

Figure 4 | Summary of spin excitations of BFA and BKFA. (a) Dispersion of spin excitations of BFA in AF phase, and BKFA in SC phase. For BKFA the reciprocal lattice units are calculated based on the orthorhombic notation with the same lattice parameters as for the AF ordered BFA. The error bars represent s.d. of the fitting (the same as for $\mathbf{b}$ and $\mathbf{c}$ ). (b,c) HWHM (damping) and integrated intensity of spin excitations of BFA and BKFA. The horizontal dotted line in $\mathbf{b}$ marks the HWHM of the total instrumental resolution of the RIXS experiment ( $40 \mathrm{meV}$ ).

cuprates differ markedly in their Fermi-surface topology, pairing symmetry and localization of the electronic bands, similar behaviour in the high-energy spin excitations upon doping highly suggests the presence of pronounced short-range spin correlations in the Fe pnictides SC phase. Interestingly, similar fluctuating magnetic moments as in cuprates were recently revealed by INS measurements on electron-doped $\mathrm{BaFe}_{1.9} \mathrm{Ni}_{0.1} \mathrm{As}_{2}$, thereby indicating the importance of strong spin correlations for Fe-pnictide superconductors ${ }^{34}$. Our RIXS study proves that the strong coupling scenario, for which the magnetic fluctuations originate from a correlated spin state, is applicable for Fe-pnictide superconductors ${ }^{7,14,15}$.

\section{Methods}

Samples and experiments. The high-quality single crystals of BFA and BKFA used in the current study were grown by the flux method as described in Chen et al. ${ }^{22}$ and Zhang et al. ${ }^{23}$ High-resolution RIXS experiments were performed using the SAXES spectrometer at the Advanced Resonant Spectroscopy (ADRESS) beamline of the Swiss Light Source, Paul Scherrer Institut, Switzerland. The energy and momentum resolutions were $40 \mathrm{meV}$ (HWHM) and $0.01 \AA^{-1}$, respectively. Samples were cleaved in situ and measured in a working vacuum better than $5 \cdot 10^{-10}$ mbar. All samples were aligned with the surface normal $(001)$ in the scattering plane. X-ray absorption was measured using the total electron yield method by recording the drain current from the samples. For RIXS measurements, linear polarized X-rays were used with the incident energy tuned to $708 \mathrm{eV}$ at the $\mathrm{L}_{3}$-edge resonance of the Fe X-ray absorption spectrum. The absolute value of the total momentum transfer, $|\mathbf{q}|$, was constant, because the scattering angle has been kept fixed at $130^{\circ}$. Momentum transfer in the a-b plane was sampled through changing the grazing incident angle. This procedure is justified by the negligible influence of the out-of-plane superexchange on the in-plane spin excitation dispersion (see Supplementary Fig. S5). All RIXS spectra were normalized to the integrated Fe 3d fluorescence intensity. The RIXS results have been reproduced for at least two samples of both parent and doped materials, respectively.

Fe 3d fluorescence background and quasi-elastic peak subtraction. In order to fit the $\mathrm{Fe} 3 \mathrm{~d}$ fluorescence line, we applied the procedure introduced in the analysis of RIXS spectra from a FeTe compound with similar fluorescence contribution ${ }^{24}$ :

$$
I_{\text {fluo }}=I_{0}\left[\alpha e^{-\mathrm{a} \omega} \omega\left(1-g_{\Gamma_{1}}\right)+\beta e^{\mathrm{b} \omega} g_{\Gamma_{1}}+\gamma e^{\mathrm{c} \omega} g_{\Gamma_{2}}\right]
$$

In the above formula, $\omega$ is the energy transfer, $\alpha e^{-\mathrm{a} \omega}$ represents the slope of the energy region above $-1 \mathrm{eV}, \beta e^{\mathrm{b} \omega}$ and $\gamma e^{\mathrm{c} \omega}$ are exponential tails, whereas $g_{\Gamma_{1,2}}=\left(e^{-\left(\omega-\omega_{1,2}\right) / \Gamma_{1,2}}+1\right)^{-1}$ gives rise to a smooth cross-over from the quasilinear to the exponential region with a width $\Gamma_{1,2}$ at the energy $\omega_{1,2}$. The quasielastic peak is fitted with two energy resolution limited Gaussian functions (Supplementary Fig. S2) accounting for elastic and phonon contributions.

Fitting of the spin excitations. The spin-excitation spectra in RIXS are fitted using the imaginary part of the system's spin susceptibility $\chi^{\prime \prime}(q, \omega)$ (ref. 11). Because the spin excitations of the parent BFA are intrinsically broadened by the finite lifetime ${ }^{30}$, we use for the RIXS fitting an asymmetrical Lorentzian function convoluted by the Gaussian resolution function:

$$
\chi^{\prime \prime}(q, \omega)=\left[\frac{\Gamma_{\mathrm{q}}}{\left(\omega-\omega_{\mathrm{q}}\right)^{2}+\Gamma_{\mathrm{q}}^{2}}-\frac{\Gamma_{\mathrm{q}}}{\left(\omega+\omega_{\mathrm{q}}\right)^{2}+\Gamma_{\mathrm{q}}^{2}}\right]
$$

In the above, $\omega_{\mathrm{q}}$ and $\Gamma_{\mathrm{q}}$ stand for the peak energy and half width at half maximum of the spin excitation (that is, damping term), respectively. This formula is also employed for the fitting of the spin-excitation data from BKFA. Examples of the fitting procedure are given in Supplementary Fig. S2.

Normalization and integration of spin excitations. All RIXS spectra are normalized to the integrated Fe $3 \mathrm{~d}$ fluorescence intensity in an energy-transfer window of -8 to $-1 \mathrm{eV}$. As the incident energy is always fixed at the $\mathrm{Fe}_{3}$ resonance, the $\mathrm{Fe} 3 \mathrm{~d}$ fluorescence intensity can be used as reference for the normalization. For the integration of the spin-excitation spectral weight, we use an energy-transfer window of -0.6 to $0.0 \mathrm{eV}$ for all RIXS spectra. Below $-0.6 \mathrm{eV}$, the spectral weight from the spin excitations is negligible.

Calculation of the spin-excitation dispersion curve. For understanding the momentum dispersion of the spin excitations in the RIXS spectra, we compare the spin-excitation dispersion curve of the INS data of a BFA parent compound ${ }^{30}$ with our RIXS results. The spin-dispersion curve from INS is reproduced using the same Heisenberg Hamiltonian consisting of effective in-plane nearest-neighbour $\left(J_{1 \mathrm{a}}\right.$ and $\left.J_{1 \mathrm{~b}}\right)$, next-nearest-neighbour $\left(J_{2}\right)$ and out-of-plane $\left(J_{\mathrm{c}}\right)$ exchange interactions as in Zhao et al. ${ }^{9}$ The dispersion relations are given by: $\mathrm{E}\left(\mathrm{q}_{/ /}\right)=\sqrt{A_{\mathbf{q}_{/ /}}^{2}-B_{\mathrm{q}_{/ /}}^{2}}$, where

$$
\begin{gathered}
A_{\mathbf{q}_{/ /}}=2 S\left[J_{1 \mathrm{~b}}(\cos (\pi K)-1)+J_{1 \mathrm{a}}+J_{\mathrm{c}}+2 J_{2}+J_{\mathrm{s}}\right], \\
B_{\mathrm{q}_{/ /}}=2 S\left[J_{\mathrm{la}} \cos (\pi H)+2 J_{2} \cos (\pi H) \cos (\pi K)+J_{\mathrm{c}} \cos (\pi L)\right]
\end{gathered}
$$

In the above relations, $J_{S}$ is the single ion anisotropy constant and $\mathbf{q} / /$ is the reduced momentum transfer away from the AF zone center $(1,0,1)$. $(\mathrm{H}, \mathrm{K}, \mathrm{L})$ is defined as $\left(\mathrm{q}_{\mathrm{x}} \mathrm{a} / 2 \pi, \mathrm{q}_{\mathrm{y}} \mathrm{b} / 2 \pi, \mathrm{q}_{\mathrm{z}} \mathrm{c} / 2 \pi\right)$ in which $\mathrm{a}=5.62 \AA, \mathrm{b}=5.57 \AA$ and $\mathrm{c}=12.97 \AA$ are the orthorhombic unit cell-lattice parameters in the spin-density wave phase. To calculate the dispersion curve for the momentum space covered in our RIXS experiment, we use the fitted exchange values from Harriger et al. ${ }^{30}$ $\mathrm{SJ}_{1 \mathrm{a}}=59.2 \pm 2.0, \mathrm{SJ}_{1 \mathrm{~b}}=-9.2 \pm 1.2, \mathrm{SJ}_{2}=13.6 \pm 1.0, \mathrm{SJ}_{\mathrm{c}}=1.8 \pm 0.3 \mathrm{meV}$ and $J_{\mathrm{s}}=1.0 . L$ is fixed to 1.0 as only negligible dispersion contributes at the center for other $L$ values. We also take into account the twinning of domains in obtaining the dispersion curves along both directions. As is shown in the main text, our RIXS data are in excellent agreement with the spin-excitation dispersion curve obtained from INS within error bars ${ }^{30}$.

When sampling the in-plane momentum transfer $q_{/ /}$by varying the incidence angle only, the out-of-plane value $\mathrm{q}_{\mathrm{perp}}$ is also changing. However, we verified in our analysis that the influence of $\mathrm{q}_{\text {perp }}$ on the in-plane spin wave dispersion shows no difference at the boundaries and only a very small $(<10 \mathrm{meV})$ dispersion at the center, which is negligible compared with our energy resolution (see Supplementary Fig. S5). 


\section{References}

1. Kamihara, Y., Watanabe, T., Hirano, M. \& Hosono, H. Iron-based layered superconductor $\mathrm{La}\left[\mathrm{O}_{1-\mathrm{x}} \mathrm{F}_{\mathrm{x}}\right] \mathrm{FeAs}(\mathrm{x}=0.05-0.12)$ with $\mathrm{Tc}=26 \mathrm{~K}$. J. Am. Chem. Soc. 130, 3296-3297 (2008).

2. Boeri, L., Dolgov, O. V. \& Golubov, A. A. Is $\mathrm{LaFeAsO}_{1-\mathrm{x}} \mathrm{F}_{\mathrm{x}}$ an electron-phonon superconductor? Phys. Rev. Lett. 101, 026403 (2008).

3. Kontani, H. \& Onari, S. Orbital-fluctuation-mediated superconductivity in iron pnictides: analysis of the filve-orbital Hubbard-Holstein model. Phys. Rev. Lett. 104, 157001 (2010).

4. Shimojima, T. et al. Orbital-independent superconducting gaps in iron pnictides. Science 332, 564 (2011).

5. Mazin, I. I., Singh, D. J., Johannes, M. D. \& Du, M. H. Unconventional superconductivity with a sign reversal in the order parameter of $\mathrm{LaFeAsO}_{1-\mathrm{x}} \mathrm{F}_{\mathrm{x}}$. Phys. Rev. Lett. 101, 057003 (2008).

6. Singh, D. J. \& Du, M. -H. Density functional study of $\mathrm{LaFeAsO}_{1-\mathrm{x}} \mathrm{F}_{\mathrm{x}}$ : a low carrier density superconductor near itinerant magnetism. Phys. Rev. Lett. 100, 237003 (2008)

7. Si, Q. \& Abrahams, E. Strong correlations and magnetic frustration in the High Tc iron pnicides. Phys. Rev. Lett. 101, 076401 (2008).

8. de la Cruz, C. et al. Magnetic order close to superconductivity in the iron-based layered $\mathrm{LaO}_{1-\mathrm{x}} \mathrm{F}_{\mathrm{x}} \mathrm{FeAs}$ systems. Nature 453, 899-902 (2008).

9. Zhao, J. et al. Spin waves and magnetic exchange interactions in $\mathrm{CaFe}_{2} \mathrm{As}_{2}$. Nat. Phys. 5, 555-560 (2009).

10. Lipscombe, O. J. et al. Spin waves in the $(\pi, 0)$ magnetically ordered iron chalcogenide $\mathrm{Fe}_{1.05}$ Te. Phys. Rev. Lett. 106, 057004 (2011).

11. Le Tacon, M. et al. Intense paramagnon excitations in a large family of hightemperature superconductors. Nat. Phys. 7, 725-730 (2011).

12. Chubukov, A. V., Efremov, D. V. \& Eremin, I. Magnetism, superconductivity, and pairing symmetry in iron-based superconductors. Phys. Rev. B 78, 134512 (2008).

13. Cvetkovic, V. \& Tesanovic, Z. Multiband magnetism and superconductivity in Fe-based compounds. Europhys. Lett. 85, 37002 (2009).

14. Hansmann, P. et al. Dichotomy between large local and small ordered magnetic moments in iron-based superconductors. Phys. Rev. Lett. 104, 197002 (2010).

15. Yin, Z. P., Haule, K. \& Kotliar, G. Kinetic frustration and the nature of the magnetic and paramagnetic states in iron pnictides and iron chalcogenides. Nat. Mater. 10, 932-935 (2011).

16. Schlappa, J. et al. Collective magnetic excitations in the spin ladder $\mathrm{Sr}_{14} \mathrm{Cu}_{24} \mathrm{O}_{41}$ measured using High-resolution resonant inelastic X-ray scattering. Phys. Rev. Lett. 103, 047401 (2009).

17. Schlappa, J. et al. Spin-orbital separation in the quasi-one-dimensional Mott insulator $\mathrm{Sr}_{2} \mathrm{CuO}_{3}$. Nature 485, 82-85 (2012).

18. Guarise, M. et al. Measurement of magnetic excitations in the two-dimensional antiferromagnetic $\mathrm{Sr}_{2} \mathrm{CuO}_{2} \mathrm{Cl}_{2}$ insulator using resonant $\mathrm{X}$-ray scattering: Evidence for extended interactions. Phys. Rev. Lett. 105, 157006 (2010).

19. Dean, M. et al. High-energy magnetic excitations in $\mathrm{Bi}_{2} \mathrm{Sr}_{2} \mathrm{CaCu}_{2} \mathrm{O}_{8}+\delta$ : Towards a unified description of the electronic and magnetic degress of freedom in the cuprates. arXiv:1212:3714.

20. Braicovich, L. et al. Magnetic excitations and phase separation in the underdoped $\mathrm{La}_{2-\mathrm{x}} \mathrm{Sr}_{\mathrm{x}} \mathrm{CuO}_{4}$ superconductor measured by resonant inelastic X-ray scattering. Phys. Rev. Lett. 104, 077002 (2010).

21. Ament, L. J. P. et al. Theoretical demonstration of how the dispersion of magnetic excitations in cuprate compounds can be determined using resonant inelastic X-ray scattering. Phys. Rev. Lett. 103, 117003 (2009).

22. Chen, G. F. et al. Transport and anisotropy in single-crystalline $\mathrm{SrFe}_{2} \mathrm{As}_{2}$ and $\mathrm{A}_{0.6} \mathrm{~K}_{0.4} \mathrm{Fe}_{2} \mathrm{As}_{2}(\mathrm{~A}=\mathrm{Sr}, \mathrm{Ba})$ superconductors. Phys. Rev. B 78, 224512 (2008).
23. Zhang, C. L. et al. Neutron scattering studies of spin excitations in hole-doped $\mathrm{Ba}_{0.67} \mathrm{~K}_{0.33} \mathrm{Fe}_{2} \mathrm{As}_{2}$ superconductor Sci. Rep. 1, 115 (2011).

24. Yang, W. L. et al. Evidence for weak electronic correlations in iron pnictides. Phys. Rev. B 80, 014508 (2009).

25. Hancock, J. N. et al. Evidence for core-hole-mediated inelastic X-ray scattering from metallic $\mathrm{Fe}_{1.087}$ Te. Phys. Rev. B 82, 020513(R) (2010).

26. Kaneshita, E., Tsutsui, K. \& Tohyama, T. Spin and orbital characters of excitations in iron arsenide superconductors revealed by simulated resonant inelastic X-ray scattering. Phys. Rev. B 84, 020511(R) (2011).

27. Fink, J., Thirupathaiah, S. \& Ovsyannikov, R. Electronic structure studies of $\mathrm{BaFe}_{2} \mathrm{As}_{2}$ by angle-resolved photoemission spectroscopy. Phys. Rev. B 79, 155118 (2009).

28. Ament, L. J. P. et al. Resonant inelastic X-ray scattering studies of elementary excitations. Rev. Mod. Phys. 83, 705-767 (2011).

29. Haverkort, M. W. Theory of resonant inelastic X-ray scattering by collective magnetic excitations. Phys. Rev. Lett. 105, 167404 (2010).

30. Harriger, L. W. et al. Nematic spin fluid in the tetragonal phase of $\mathrm{BaFe}_{2} \mathrm{As}_{2}$ Phys. Rev. B 84, 054544 (2011).

31. Li, H.-F. et al. Anisotropic and quasipropagating spin excitations in superconducting $\mathrm{Ba}\left(\mathrm{Fe}_{0.926} \mathrm{Co}_{0.074}\right)_{2} \mathrm{As}_{2}$. Phys. Rev. B 82, 140503(R) (2010).

32. Hayden, S. M. et al. Comparison of the high-frequency magnetic fluctuations in insulating and superconducting $\mathrm{La}_{2-\mathrm{x}} \mathrm{Sr}_{\mathrm{x}} \mathrm{CuO}_{4}$. Phys. Rev. Lett. 76, 1344 (1996).

33. da Conceição, C. M. S., Silva Neto, M. B. \& Marino, E. C. Magnetic and quasiparticle excitation spectra of an Itinerant $J_{1}-J_{2}$ model for Iron-pnictide superconductors. Phys. Rev. Lett. 106, 11702 (2011).

34. Liu, M. et al. Nature of magnetic excitations in superconducting $\mathrm{BaFe}_{1.9} \mathrm{Ni}_{0.1} \mathrm{As}_{2}$. Nat. Phys. 8, 376-381 (2012).

\section{Acknowledgements}

This work was performed at the ADRESS beamline of the Swiss Light Source using the SAXES instrument jointly built by Paul Scherrer Institut, Switzerland and Politecnico d Milano, Italy. We acknowledge valuable discussion with T. Tohyama, K. Wohlfeld and M. Daghofer. We thank for financial support through the Swiss National Science Foundation and its NCCR MaNEP, as well as through the National Basic Research (973) Program of China and the Chinese Academy of Sciences.

\section{Author contributions}

K.J.Z., H.D. and T.S. conceived the project. K.J.Z., Y.B.H., C.M., V.N.S., L.P. and T.S. carried out the experiments. N.L.W., Z.G.C., C.Z. and P.D. fabricated samples. Y.B.H. and Z.G.C. conducted sample characterization. K.J.Z. and T.S. analysed experimental data. K.J.Z., J.v.d.B., H.D. and T.S. wrote the manuscript with particular input from X.D. and all other coauthors.

\section{Additional information}

Supplementary Information accompanies this paper on http://www.nature.com/ naturecommunications

Competing financial interests: The authors declare no competing financial interests.

Reprints and permission information is available online at http://npg.nature.com/ reprintsandpermissions/

How to cite this article: Zhou, K-J. et al. Persistent high-energy spin excitations in iron pnictide superconductors. Nat. Commun. 4:1470 doi: 10.1038/ncomms2428 (2013) 